\title{
The Zariski topology-graph of modules over commutative rings II
}

Received: 24 January 2020 / Accepted: 22 October 2020 / Published online: 9 November 2020

(C) The Author(s) 2020

\begin{abstract}
Let $M$ be a module over a commutative ring $R$. In this paper, we continue our study about the Zariski topology-graph $G\left(\tau_{T}\right)$ which was introduced in Ansari-Toroghy et al. (Commun Algebra 42:3283$3296,2014)$. For a non-empty subset $T$ of $\operatorname{Spec}(M)$, we obtain useful characterizations for those modules $M$ for which $G\left(\tau_{T}\right)$ is a bipartite graph. Also, we prove that if $G\left(\tau_{T}\right)$ is a tree, then $G\left(\tau_{T}\right)$ is a star graph. Moreover, we study coloring of Zariski topology-graphs and investigate the interplay between $\chi\left(G\left(\tau_{T}\right)\right)$ and $\omega\left(G\left(\tau_{T}\right)\right)$.
\end{abstract}

\section{Mathematics Subject Classfication $13 \mathrm{C} 13 \cdot 13 \mathrm{C} 99 \cdot 05 \mathrm{C} 75$}

\section{Introduction}

Throughout this paper, $R$ is a commutative ring with a non-zero identity and $M$ is a unital $R$-module. By $N \leq M$ (resp. $N<M$ ) we mean that $N$ is a submodule (resp. proper submodule) of $M$.

Define $\left(N:_{R} M\right)$ or simply $(N: M)=\{r \in R \mid r M \subseteq N\}$ for any $N \leq M$. We denote $((0): M)$ by $\operatorname{Ann}_{R}(M)$ or simply $\operatorname{Ann}(M) . M$ is said to be faithful if $\operatorname{Ann}(M)=(0)$. [3]).

Let $N, K \leq M$. Then the product of $N$ and $K$, denoted by $N K$, is defined by $(N: M)(K: M) M$ (see

A prime submodule of $M$ is a submodule $P \neq M$ such that whenever $r e \in P$ for some $r \in R$ and $e \in M$, we have $r \in(P: M)$ or $e \in P[10]$.

The prime spectrum of $M$ is the set of all prime submodules of $M$ and denoted by $\operatorname{Spec}(M)$.

If $N$ is a submodule of $M$, then $V(N)=\{P \in \operatorname{Spec}(M) \mid(P: M) \supseteq(N: M)\}$ [11].

The Zariski topology on $X=\operatorname{Spec}(M)$ is the topology $\tau_{M}$ described by taking the set $Z(M)=\{V(N) \mid$ $N$ is a submodule of $M\}$ as the set of closed sets of $\operatorname{Spec}(M)$ [11].

A topological space $X$ is irreducible if for any decomposition $X=X_{1} \cup X_{2}$ with closed subsets $X_{i}$ of $X$ with $i=1,2$, we have $X=X_{1}$ or $X=X_{2}$.

There are many papers on assigning graphs to rings or modules (see, for example, [1,5,6,9]). In [4], the present authors introduced and studied the graph $G\left(\tau_{T}\right)$ and $A G(M)$, called the Zariski topology-graph and the annihilating-submodule graph, respectively.

H. Ansari-Toroghy · S. Habibi ( $\varangle)$

Department of Pure Mathematics, Faculty of Mathematical Sciences, University of Guilan, P. O. Box 41335-19141, Rasht, Iran E-mail: habibishk@gmail.com

H. Ansari-Toroghy

E-mail: ansari@guilan.ac.ir

S. Habibi

School of Mathematics, Institute for Research in Fundamental Sciences (IPM), P.O. Box: 19395-5746, Tehran, Iran 
Let $T$ be a non-empty subset of $\operatorname{Spec}(M)$. The Zariski topology-graph $G\left(\tau_{T}\right)$ is an undirected graph with vertices $V\left(G\left(\tau_{T}\right)\right)=\{N<M \mid$ there exists $K<M$ such that $V(N) \cup V(K)=T$ and $V(N), V(K) \neq T\}$ and distinct vertices $N$ and $L$ are adjacent if and only if $V(N) \cup V(L)=T$ (see [4, Definition 2.3]).

$A G(M)$ is an undirected graph with vertices $V(A G(M))=\{N \leq M \mid$ there exists $(0) \neq K<M$ with $N K=(0)\}$. In this graph, distinct vertices $N, L \in V(A G(M))$ are adjacent if and only if $N L=(0)$. Let $A G(M)^{*}$ be the subgraph of $A G(M)$ with vertices $V\left(A G(M)^{*}\right)=\{N<M$ with $(N: M) \neq \operatorname{Ann}(M) \mid$ there exists a submodule $K<M$ with $(K: M) \neq \operatorname{Ann}(M)$ and $N K=(0)\}$. By [4, Theorem 3.4], one conclude that $A G(M)^{*}$ is a connected subgraph.

If $\operatorname{Spec}(M) \neq \emptyset$, the mapping $\psi: \operatorname{Spec}(M) \rightarrow \operatorname{Spec}(R / \operatorname{Ann}(M))$ such that $\psi(P)=(P: M) / \operatorname{Ann}(M)$ for every $P \in \operatorname{Spec}(M)$, is called the natural map of $\operatorname{Spec}(M)$ [11].

The prime radical $\sqrt{N}$ is defined to be the intersection of all prime submodules of $M$ containing $N$, and in case $N$ is not contained in any prime submodule, $\sqrt{N}$ is defined to be $M$ [10].

We recall that $N<M$ is said to be a semiprime submodule of $M$ if for every ideal $I$ of $R$ and every submodule $K$ of $M$ with $I^{2} K \subseteq N$ implies that $I K \subseteq N$. Further $M$ is called a semiprime module if (0) $\subseteq M$ is a semiprime submodule. Every intersection of prime submodules is a semiprime submodule (see [17]).

The notations $\operatorname{Nil}(R), \operatorname{Min}(M)$, and $\operatorname{Min}(T)$ will denote the set of all nilpotent elements of $R$ and the set of all minimal prime submodules of $M$, and the set of minimal members of $T$, respectively.

A clique of a graph is a complete subgraph and the supremum of the sizes of cliques in $G$, denoted by $\omega(G)$, is called the clique number of $G$. Let $\chi(G)$ denote the chromatic number of the graph $G$, that is, the minimal number of colors needed to color the vertices of $G$ so that no two adjacent vertices have the same color. Obviously $\chi(G) \geq \omega(G)$.

In this article, we continue our studying about $G\left(\tau_{T}\right)$ and $A G(M)$ and we try to relate the combinatorial properties of the above mentioned graphs to the algebraic properties of $M$.

In Sect. 2 of this paper, we state some properties related to the Zariski topology-graph that are basic or needed in the later sections. In Sect. 3, we study the bipartite Zariski topology-graphs of modules over commutative rings (see Proposition 3.1). Also, we prove that if $G\left(\tau_{T}\right)$ is a tree, then $G\left(\tau_{T}\right)$ is a star graph (see Theorem 3.5). In Sect. 4, we study coloring of the Zariski topology-graph of modules and investigate the interplay between $\chi\left(G\left(\tau_{T}\right)\right)$ and $\omega\left(G\left(\tau_{T}\right)\right)$. We show that under condition over minimal submodules of $M /\left(\cap_{P \in T} P: M\right) M$, we have $\omega\left(G\left(\tau_{T}\right)\right)=\chi\left(G\left(\tau_{T}\right)\right)$ (see Theorem 4.1). Moreover, we investigate some relations between the existence of cycles in the Zariski topology-graph of a cyclic module and the number of its minimal members of $T$ (see Proposition 4.10).

Let us introduce some graphical notions and denotations that are used in what follows: A graph $G$ is an ordered triple $\left(V(G), E(G), \psi_{G}\right)$ consisting of a nonempty set of vertices, $V(G)$, a set $E(G)$ of edges, and an incident function $\psi_{G}$ that associates an unordered pair of distinct vertices with each edge. The edge $e$ joins $x$ and $y$ if $\psi_{G}(e)=\{x, y\}$, and we say $x$ and $y$ are adjacent. A path in a graph $G$ is a finite sequence of vertices $\left\{x_{0}, x_{1}, \ldots, x_{n}\right\}$, where $x_{i-1}$ and $x_{i}$ are adjacent for each $1 \leq i \leq n$ and we denote $x_{i-1}-x_{i}$ for existing an edge between $x_{i-1}$ and $x_{i}$.

A graph $H$ is a subgraph of $G$, if $V(H) \subseteq V(G), E(H) \subseteq E(G)$, and $\psi_{H}$ is the restriction of $\psi_{G}$ to $E(H)$. A bipartite graph is a graph whose vertices can be divided into two disjoint sets $U$ and $V$ such that every edge connects a vertex in $U$ to one in $V$; that is, $U$ and $V$ are each independent sets and complete bipartite graph on $n$ and $m$ vertices, denoted by $K_{n, m}$, where $V$ and $U$ are of size $n$ and $m$, respectively, and $E(G)$ connects every vertex in $V$ with all vertices in $U$. Note that a graph $K_{1, m}$ is called a star graph and the vertex in the singleton partition is called the center of the graph. For some $U \subseteq V(G)$, we denote by $N(U)$, the set of all vertices of $G \backslash U$ adjacent to at least one vertex of $U$. For every vertex $v \in V(G)$, the size of $N(v)$ is denoted by $\operatorname{deg}(v)$. If all the vertices of $G$ have the same degree $k$, then $G$ is called $k$-regular, or simply regular. We denote by $C_{n}$ a cycle of order $n$. Let $G$ and $G^{\prime}$ be two graphs. A graph homomorphism from $G$ to $G^{\prime}$ is a mapping $\phi: V(G) \longrightarrow V\left(G^{\prime}\right)$ such that for every edge $\{u, v\}$ of $G,\{\phi(u), \phi(v)\}$ is an edge of $G^{\prime}$. A retract of $G$ is a subgraph $H$ of $G$ such that there exists a homomorphism $\phi: G \longrightarrow H$ such that $\phi(x)=x$, for every vertex $x$ of $H$. The homomorphism $\phi$ is called the retract (graph) homomorphism (see [13]).

Throughout the rest of this paper, we denote by $T$ a non-empty subset of $\operatorname{Spec}(M), F:=\cap_{P \in T} P$, $Q:=(F: M) M, \bar{M}:=M / Q, N:=N / Q, \bar{m}:=m+Q$, and $\bar{I}:=I /(Q: M)$, where $N$ is a submodule of $M$ containing $Q, m \in M$, and $I$ is an ideal of $R$ containing $(Q: M)$. 


\section{Auxiliary results}

In this section, we provide some properties related to the Zariski topology-graph that are basic or needed in the sequel.

Remark 2.1 Let $N$ be a submodule of $M$. Set $V^{*}(N):=\{P \in \operatorname{Spec}(M) \mid P \supseteq N\}$. By [4, Remark 2.2], for submodules $N$ and $K$ of $M$, we have

$$
V(N) \cup V(K)=V(N \cap K)=V(N K)=V^{*}(N K) .
$$

By [4, Remark 2.5], we have $T$ is a closed subset of $\operatorname{Spec}(M)$ if and only if $T=V(F)$ and $G\left(\tau_{T}\right) \neq \emptyset$ if and only if $T=V(F)$ and $T$ is not irreducible. So if $N$ and $K$ are adjacent in $G\left(\tau_{T}\right)$, then $V^{*}(N K)=V^{*}(Q)$ and hence $\sqrt{N K}=F$. Therefore, $F \subseteq \sqrt{(N: M) M}$ and $F \subseteq \sqrt{(K: M) M}$.

Lemma 2.2 (See [2, Proposition 7.6]) Let $R_{1}, R_{2}, \ldots, R_{n}$ be non-zero ideals of $R$. Then the following statements are equivalent:

(a) $R=R_{1} \oplus \ldots \oplus R_{n}$;

(b) As an abelian group $R$ is the direct sum of $R_{1}, \ldots, R_{n}$;

(c) There exist pairwise orthogonal idempotents $e_{1}, \ldots, e_{n}$ with $1=e_{1}+\ldots+e_{n}$, and $R_{i}=R e_{i}, i=1, \ldots, n$.

Proposition 2.3 Suppose that e is an idempotent element of $R$. We have the following statements.

(a) $R=R_{1} \oplus R_{2}$, where $R_{1}=e R$ and $R_{2}=(1-e) R$.

(b) $M=M_{1} \oplus M_{2}$, where $M_{1}=e M$ and $M_{2}=(1-e) M$.

(c) For every submodule $N$ of $M, N=N_{1} \oplus N_{2}$ such that $N_{1}$ is an $R_{1}$-submodule $M_{1}, N_{2}$ is an $R_{2}$-submodule $M_{2}$, and $\left(N:_{R} M\right)=\left(N_{1}: R_{1} M_{1}\right) \oplus\left(N_{2}: R_{2} M_{2}\right)$.

(d) For submodules $N$ and $K$ of $M, N K=N_{1} K_{1} \oplus N_{2} K_{2}, N \cap K=N_{1} \cap K_{1} \oplus N_{2} \cap K_{2}$ such that $N=N_{1} \oplus N_{2}$ and $K=K_{1} \oplus K_{2}$.

(e) Prime submodules of $M$ are $P \oplus M_{2}$ and $M_{1} \oplus Q$, where $P$ and $Q$ are prime submodules of $M_{1}$ and $M_{2}$, respectively.

(f) For submodule $N$ of $M$, we have $\sqrt{N}=\sqrt{N_{1} \oplus N_{2}}=\sqrt{N_{1}} \oplus \sqrt{N_{2}}$, where $N=N_{1} \oplus N_{2}$.

Proof This is clear.

An ideal $I<R$ is said to be nil if $I$ consist of nilpotent elements.

Lemma 2.4 (See [15, Theorem 21.28]) Let I be a nil ideal in $R$ and $u \in R$ be such that $u+I$ is an idempotent in $R / I$. Then there exists an idempotent e in $u R$ such that $e-u \in I$.

Lemma 2.5 (See [5, Lemma 2.4]) Let $N$ be a minimal submodule of $M$ and let $\operatorname{Ann}(M)$ be a nil ideal. Then we have $N^{2}=(0)$ or $N=e M$ for some idempotent $e \in R$.

We note that $M$ is said to be primeful if either $M=(0)$ or $M \neq(0)$ and the natural map of $\operatorname{Spec}(M)$ is surjective (see [12]).

Proposition 2.6 We have the following statements.

(a) If $N, L$ are adjacent in $G\left(\tau_{T}\right)$, then $\sqrt{(N: M) M} / F$ and $\sqrt{(L: M) M} / F$ are adjacent in $A G(M / F)$.

(b) If $M$ is a primeful module and $N, L$ are adjacent in $G\left(\tau_{T}\right)$, then $\sqrt{N} / F$ and $\sqrt{L} / F$ are adjacent in $A G(M / F)$.

Proof (a) First, we see easily that for any submodule $N$ of $M, V(N)=V(\sqrt{(N: M) M})$. Suppose that $N$ and $L$ are adjacent in $G\left(\tau_{T}\right)$ so that $V(N) \cup V(L)=T$. Then we have $V^{*}(\sqrt{(N: M) M} \sqrt{(L: M) M})=T$. It follows that $\sqrt{(N: M) M} \sqrt{(L: M) M} \subseteq F$ (see Remark 2.1). Also by Remark 2.1, $F \subseteq \sqrt{(N: M) M}$ and $F \subseteq \sqrt{(L: M) M}$. Therefore, $\sqrt{(N: M) M} / F$ and $\sqrt{(L: M) M} / F$ are adjacent in $A G(M / F)$.

(b) This is clear by [4, Corollary 4.5].

Remark 2.7 The Proposition 2.6(a) extends [4, Theorem 4.4].

Lemma 2.8 Assume that $T$ is a closed subset of $\operatorname{Spec}(M)$. Then $A G(\bar{M})^{*}$ is isomorphic with a subgraph of $G\left(\tau_{T}\right)$. In particular, $A G(M / F)^{*}$ is isomorphic with an induced subgraph of $G\left(\tau_{T}\right)$. 
Proof Let $\bar{N} \in V\left(A G(\bar{M})^{*}\right)$. Then there exists a nonzero submodule $\bar{K}$ of $\bar{M}$ such that it is adjacent to $\bar{N}$ (if $N=K$, then $(N: M)=(Q: M)$, a contradiction). So we have $N K \subseteq Q$. Hence $V(N K) \supseteq T$. Since $Q \subseteq N$ and $Q \subseteq K$, then $V(N) \subseteq T$ and $V(K) \subseteq T$. Therefore $V(N K)=T$ (if $V(N)=T$, then $(N: M)=(Q: M)$, a contradiction). Hence $N$ is a vertex in $G\left(\tau_{T}\right)$ which is adjacent to $K$. To see the last assertion, let $N / F$ and $K / F$ be two vertices of $A G(M / F)^{*}$. If $N$ and $K$ are adjacent in $G\left(\tau_{T}\right)$, then by Proposition 2.6, $\sqrt{(N: M) M} / F$ and $\sqrt{(K: M) M} / F$ are adjacent in $A G(M / F)$. So

$$
\sqrt{(N: M) M} \sqrt{(K: M) M} \subseteq F .
$$

Since

$$
N K=((N: M) M: M)((K: M) M: M) M \subseteq \sqrt{(N: M) M} \sqrt{(K: M) M},
$$

we have $N / F$ and $K / F$ are adjacent in $A G(M / F)^{*}$, as desired.

Lemma 2.9 If $\bar{M}$ is a faithful module and $T$ is a closed subset of $\operatorname{Spec}(M)$, then $G\left(\tau_{\operatorname{Spec}(M)}\right)$ and $A G(M)^{*}$ are the same.

Proof $\bar{M}$ is a faithful module and $T$ is a closed subset of $\operatorname{Spec}(M)$ so that $T=\operatorname{Spec}(M)$. If $G\left(\tau_{\operatorname{Spec}(M)}\right) \neq \emptyset$, then there exist non-trivial submodules $N$ and $K$ of $M$ which are adjacent in $G\left(\tau_{\operatorname{Spec}(M)}\right)$. Hence $V(N K)=$ $\operatorname{Spec}(M)$ which implies that $N K=(0)$ so that $A G(M)^{*} \neq \emptyset$. By Lemma 2.8, $A G(M)^{*}$ is isomorphic with a subgraph of $G\left(\tau_{\operatorname{Spec}(M)}\right)$. One can see that the vertex map $\phi: V\left(G\left(\tau_{\operatorname{Spec}(M)}\right)\right) \longrightarrow V\left(A G(M)^{*}\right)$, defined by $N \longrightarrow N$ is an isomorphism.

Recall that $\Delta\left(G\left(\tau_{T}\right)\right)$ is the maximum degree of $G\left(\tau_{T}\right)$ and the length of an $R$-module $M$, is denoted by $l_{R}(M)$.

Lemma 2.10 Let every nontrivial submodule of $M$ be a vertex in $G\left(\tau_{T}\right)$. If $\Delta\left(G\left(\tau_{T}\right)\right)<\infty$, then $l_{R}(M) \leq$ $\Delta\left(G\left(\tau_{T}\right)\right)+1$. Also, every non-trivial submodule of $M$ has finitely many submodules.

Proof First, we show that the descending chain of non-trivial submodules $K_{1} \supsetneq K_{2} \supsetneq K_{3} \supsetneq \ldots$ terminates. Since $G\left(\tau_{T}\right)$ is connected, there exists a submodule $N$ such that $V(N) \cup V\left(K_{1}\right)=T$. Hence for each $i$, $i \geq 1, V(N) \cup V\left(K_{i}\right)=T$ and so $\operatorname{deg}(N)=\infty$, a contradiction. Next, let $N_{1} \subsetneq N_{2} \subsetneq N_{3} \subsetneq \ldots$ be an ascending chain of non-trivial submodules of $M$. Since $G\left(\tau_{T}\right)$ is connected, there exists a submodule $K$ such that $V(K) \cup V\left(N_{\Delta+1}\right)=T$, where $\Delta=\Delta\left(G\left(\tau_{T}\right)\right)$. Hence $V(K) \cup V\left(N_{i}\right)=T$ for each $1 \leq i \leq \Delta+1$. Thus $\operatorname{deg}(K) \geq \Delta+1$, a contradiction. It follows that $l_{R}(M) \leq \Delta+1$. For the proof of the last assertion, let $N$ be a non-trivial submodule of $M$. Since $G\left(\tau_{T}\right)$ is connected, there exists a submodule $K$ such that $V(N) \cup V(K)=T$. Hence for every submodule $N^{\prime}$ of $N, V\left(N^{\prime}\right) \cup V(K)=T$. As $\Delta<\infty$, the number of submodules of $N$ should be finite.

Theorem 2.11_Suppose that $\bar{M}$ is a multiplication module and $G\left(\tau_{T}\right) \neq \emptyset$. If $G\left(\tau_{T}\right)$ has acc (resp. dcc) on vertices, then $\bar{M}$ is a Noetherian (resp. an Artinian) module.

Proof Suppose that $G\left(\tau_{T}\right)$ has acc (resp. dcc) on vertices. By [4, Remark 2.6], $F$ is not a prime submodule of $M$ and hence there exist $r \in R$ and $m \in M$ such that $r m \in F$ but $m \notin F$ and $r \notin(F: M)$. Now $\overline{r M} \cong \bar{M} /(\overline{0}: \bar{M} r)$. Further, $\overline{r M}$ and $(\overline{0}: \bar{M} r)$ are vertices in $A G(\bar{M})=A G(\bar{M})^{*}(\bar{M}$ is a multiplication module) because $(\overline{0}: \bar{M} r)(\overline{r M})=((\overline{0}: \bar{M} r): \bar{M})(\overline{r M}: \bar{M}) \bar{M} \subseteq \overline{r M}((\overline{0}: \bar{M} r): \bar{M}) \subseteq r(\overline{0}: \bar{M} r)=\overline{0}$. Then by Lemma 2.8, $\left\{N \mid \bar{N} \leq \bar{M}, \bar{N} \subseteq \frac{\bar{M}}{r M}\right\} \cup\{N \mid \bar{N} \leq \bar{M}, \bar{N} \subseteq(\overline{0}: \bar{M} r)\} \subseteq V\left(G\left(\tau_{T}\right)\right)$. It follows that the $R$-modules $\overline{r M}$ and $(\overline{0}: \bar{M} r)$ have acc (resp. dcc) on submodules. Since $\overline{r M} \cong \bar{M} /(\overline{0}: \bar{M} r), \bar{M}$ has acc on submodules and the proof is completed.

\section{Zariski topology-graph of modules}

First, in this section we give the more notation to be used throughout the remainder of this article. Suppose that $e$ $(e \neq 0,1)$ is an idempotent element of $R$. Let $M_{1}:=e M, M_{2}:=(1-e) M, T_{1}:=\left\{P_{1} \in \operatorname{Spec}\left(M_{1}\right) \mid P_{1} \oplus M_{2} \in\right.$ $T\}, T_{2}:=\left\{P_{2} \in \operatorname{Spec}\left(M_{2}\right) \mid M_{1} \oplus P_{2} \in T\right\}, F_{1}:=\cap_{P_{1} \in T_{1}} P_{1}, F_{2}:=\cap_{P_{2} \in T_{2}} P_{2}, Q_{1}:=\left(F_{1}: M_{1}\right) M_{1}, Q_{2}:=$ $\left(F_{2}: M_{2}\right) M_{2}, \bar{M}_{1}=\overline{e M}=e M / Q_{1}$, and $\bar{M}_{2}=\overline{(e-1) M}=(e-1) M / Q_{2}$. Consequently, we have, $Q=Q_{1} \oplus Q_{2}$, where $Q=\left(\cap_{P \in T} P: M\right) M$ and $\bar{M} \cong \bar{M}_{1} \oplus \bar{M}_{2}$

We recall that a submodule $N$ of $M$ is a prime $R$-module if and only if it is a prime $R / \operatorname{Ann}(M)$-module (see [16, Result 1.2]). 
Proposition 3.1 Suppose that $\bar{M}$ does not have a non-zero submodule $\bar{F} \neq \bar{N}$ with $V(N)=T$ and $\operatorname{Ann}(\bar{M})$ is a nil ideal. Then the following statements hold.

(a) If there exists a vertex of $G\left(\tau_{T}\right)$ which is adjacent to every other vertex, then $\bar{M}_{1}$ is a simple module and $\bar{M}_{2}$ is a prime module for some idempotent element $e \in R$.

(b) If $\bar{M}_{1}$ and $\bar{M}_{2}$ are prime modules for some idempotent element $e \in R$, then $G\left(\tau_{T}\right)$ is a complete bipartite graph.

Proof - (a) Suppose that $N$ is adjacent to every other vertex of $G\left(\tau_{T}\right)$. Since $V(N)=V((N: M) M)$, we have $N=(N: M) M$ and hence $V(N)=V^{*}(N)$. Thus, $N=\sqrt{N}$ because $V(N)=V(\sqrt{N})$. We claim that $\bar{N}$ is a minimal submodule of $\bar{M}$. Let $Q \subsetneq K \subsetneq N$. If $V(K) \neq T$, then $K$ is adjacent to $N$ and hence $V(K)=T$, a contradiction. So $\bar{N}$ is a minimal submodule of $\bar{M}$. We have $(\bar{N})^{2} \neq(0)$ because $V(N) \neq T$. Then Lemma 2.5, implies that $\bar{M} \cong \overline{e M} \oplus \overline{(e-1) M}$ for some idempotent element $e$ of $R$. Without loss of generality we may assume that $M_{1} \oplus Q_{2}$ is adjacent to every other vertex. Since $V\left(F_{1} \oplus Q_{2}\right)=V\left(Q_{1} \oplus F_{2}\right)=T$, the assumption of theorem implies that $F=Q$. We claim that $\bar{M}_{1}$ is a simple module and $\bar{M}_{2}$ is a prime module. Let $Q_{1} \subsetneq K<M_{1}$. We have $V\left(K \oplus Q_{2}\right) \neq T$ because $Q_{1} \oplus Q_{2} \subsetneq K \oplus Q_{2}$. Since $V\left(K \oplus Q_{2}\right) \cup V\left(Q_{1} \oplus M_{2}\right)=T$, we have $K \oplus Q_{2}$ is a vertex and hence is adjacent to $M_{1} \oplus Q_{2}$. Therefore $V\left(K \oplus Q_{2}\right) \cup V\left(M_{1} \oplus Q_{2}\right)=V\left(K \oplus Q_{2}\right)=T$, a contradiction. It implies that $\bar{M}_{1}$ is a simple module. Now, we show that $\bar{M}_{2}$ is a prime module. It is enough to show that it is a prime $R /\left(Q_{2}: M_{2}\right)$-module. Otherwise, $\bar{I} \bar{K}=(\overline{0})$, where $\left(Q_{2}: M_{2}\right) \subsetneq I<R$ and $Q_{2} \subsetneq K<M_{2}$. It follows that $V\left(M_{1} \oplus K\right) \cup V\left(Q_{1} \oplus I M_{2}\right)=V\left(Q_{1} \oplus K\left(I M_{2}\right)\right)=T$ because $K\left(I M_{2}\right) \subseteq I K \subseteq Q_{2}$ (note that $\left(Q_{2}: M_{2}\right) \subseteq\left(K: M_{2}\right)$ and $\left.\left(Q_{2}: M_{2}\right) \subseteq I\right)$. Therefore, $M_{1} \oplus K$ is a vertex and hence is adjacent to $M_{1} \oplus Q_{2}$. So $V\left(M_{1} \oplus K\right) \cup V\left(M_{1} \oplus Q_{2}\right)=T=V\left(M_{1} \oplus Q_{2}\right)$, a contradiction (note that $M_{1} \oplus K$ is properly containing $Q_{1} \oplus Q_{2}$ ).

- (b) Assume that $N_{1} \oplus N_{2}$ is adjacent to $K_{1} \oplus K_{2}$. One can see that $\sqrt{N_{1} K_{1}} \oplus \sqrt{N_{2} K_{2}}=\sqrt{Q_{1}} \oplus \sqrt{Q_{2}}$. It implies that $\overline{\left(\sqrt{\left(K_{1}: M_{1}\right) M_{1}}: M_{1}\right)} \overline{\sqrt{\left(N_{1}: M_{1}\right) M_{1}}}=(\overline{0})$ and $\overline{\left(\sqrt{\left(K_{2}: M_{2}\right) M_{2}}: M_{2}\right)} \frac{\sqrt{\left(N_{2}: M_{2}\right) M_{2}}}{\sqrt{(0)}}=$ $(\overline{0})$. Since $\bar{M}_{1}$ and $\bar{M}_{2}$ are prime modules, $\left(\sqrt{\left(K_{1}: M_{1}\right) M_{1}}: M_{1}\right)=\left(Q_{1}: M_{1}\right)$ or $\sqrt{\left(N_{1}: M_{1}\right) M_{1}}=Q_{1}$ and $\left(\sqrt{\left(K_{2}: M_{2}\right) M_{2}}: M_{2}\right)=\left(Q_{2}: M_{2}\right)$ or $\sqrt{\left(N_{2}: M_{2}\right) M_{2}}=Q_{2}$. Therefore $G\left(\tau_{T}\right)$ is a complete bipartite graph with two parts $U$ and $V$ such that $N \in U$ if and only if $V(N)=V\left(M_{1} \oplus Q_{2}\right)$ and $K \in V$ if and only if $V(K)=V\left(Q_{1} \oplus M_{2}\right)$.

Corollary 3.2 Let $\bar{M}$ be a faithful module which does not have a non-zero submodule $\bar{F} \neq \bar{N}$ with $V(N)=T$. Then the following statements are equivalent.

(a) There is a vertex of $G\left(\tau_{\operatorname{Spec}(M)}\right)$ which is adjacent to every other vertex of $G\left(\tau_{\operatorname{Spec}(M)}\right)$.

(b) $G\left(\tau_{\operatorname{Spec}(M)}\right)$ is a star graph.

(c) $M=F \oplus D$, where $F$ is a simple module and $D$ is a prime module.

Proof (a) $\Rightarrow$ (b) Let $\bar{M}$ be a faithful module. Then $Q=(0)$ and we have $T=\operatorname{Spec}(M)$. By Proposition 3.1, $M=M_{1} \oplus M_{2}$, where $M_{1}$ is a simple module and $M_{2}$ is a prime module. Then every non-zero submodule of $M$ is of the form $M_{1} \oplus N_{2}$ and $(0) \oplus N_{2}$, where $N_{2}$ is a non-zero submodule of $M_{2}$. We show that non of the submodules of the form (0) $\oplus N_{2}$ can be adjacent to each other. Assume that (0) $\oplus N_{2}$ and (0) $\oplus K_{2}$ are adjacent in $G\left(\tau_{\operatorname{Spec}(M)}\right)$, where $(0) \neq N_{2} \leq M_{2}$ and $(0) \neq K_{2} \leq M_{2}$. Since (0) is a prime submodule of $M_{2}$, by Remark 2.1, we have $N_{2} K_{2}=(0)$. Hence $V\left((0) \oplus N_{2}\right)=\operatorname{Spec}(M)$ or $V\left((0) \oplus K_{2}\right)=\operatorname{Spec}(M)$, a contradiction. Similarly, we can not have any vertex of the form $M_{1} \oplus N_{2}$, where $N_{2}$ is a non-zero proper submodule of $M_{2}$. Now it is easy to see that $M_{1} \oplus(0)$ is adjacent to every other vertex and so $G\left(\tau_{\operatorname{Spec}(M)}\right)$ is a star graph.

(b) $\Rightarrow$ (c) This follows by Proposition 3.1(a).

(c) $\Rightarrow$ (a) Assume that $M=F \oplus D$, where $F$ is a simple module and $D$ is a prime module. Using the Proposition 3.1 (b), $G\left(\tau_{\operatorname{Spec}(M)}\right)$ is a complete bipartite graph with two parts $U$ and $V$ such that $N \in U$ if and only if $V(N)=V(F \oplus(0))$ and $K \in V$ if and only if $V(K)=V((0) \oplus D)$. We claim that $|U|=1$. Otherwise, $V(F \oplus(0))=V(N \oplus K)$, where $N=(0)$ or $N=F$ and $(0) \neq K<D$. Therefore $V(N \oplus K) \cup V((0) \oplus D)=$ $\operatorname{Spec}(M)$ and hence $V((0) \oplus K)=\operatorname{Spec}(M)$ that is a contradiction with our assumption. So $F \oplus(0)$ is adjacent to every other vertex of $G\left(\tau_{\operatorname{Spec}(M)}\right)$

Lemma 3.3 Let $\in R$ be an idempotent element of $R$ and suppose that $\bar{M}$ does not have a non-zero submodule $\bar{F} \neq \bar{N}$ with $V(N)=T$. If $G\left(\tau_{T}\right)$ is a triangle-free graph, then both $\bar{M}_{1}$ and $\bar{M}_{2}$ are prime $R$-modules. Moreover, if $G\left(\tau_{T}\right)$ has no cycle, then $\bar{M}_{1}$ is a simple module and $\bar{M}_{2}$ is a prime module. 
Proof First recall that if $\bar{M}$ does not have a non-zero submodule $\bar{F} \neq \bar{N}$ with $V(N)=T$, then $F=Q$ because $V\left(F_{1} \oplus Q_{2}\right)=V\left(Q_{1} \oplus F_{2}\right)=T$. Without loss of generality, we can assume that $\bar{M}_{1}$ is not a prime module. Then $\bar{I} \bar{K}=(\overline{0})$, where $\left(Q_{1}: M_{1}\right) \subsetneq I<R$ and $Q_{1} \subsetneq K<M_{1}$. It follows that $Q_{1} \oplus M_{2}, K \oplus Q_{2}$, and $I M_{1} \oplus Q_{2}$ form a triangle in $G\left(\tau_{T}\right)$, a contradiction (note that $V\left(K \oplus Q_{2}\right) \cup V\left(I M_{1} \oplus Q_{2}\right)=V\left(K\left(I M_{1}\right) \oplus Q_{2}\right)=T$. Also $I M_{1} \neq K$. Otherwise, $V\left(K \oplus Q_{2}\right)=V\left(K^{2} \oplus Q_{2}\right)=V\left(K\left(I M_{1}\right) \oplus Q_{2}\right)=T$, a contradiction). So both $\bar{M}_{1}$ and $\bar{M}_{2}$ are prime $R$-modules. Now suppose that $G\left(\tau_{T}\right)$ has no cycle. If none of $\bar{M}_{1}$ and $\bar{M}_{2}$ is a simple module, then we choose non-trivial submodules $N_{i}$ in $M_{i}$ for some $i=1$, 2. So $N_{1} \oplus Q_{2}, Q_{1} \oplus N_{2}, M_{1} \oplus Q_{2}$, and $Q_{1} \oplus M_{2}$ form a cycle, a contradiction.

Corollary 3.4 Assume that $M$ is a multiplication module or a primeful module, $\operatorname{Ann}(\bar{M})$ is a nil ideal, and $\bar{M}$ does not have a non-zero submodule $\bar{F} \neq \bar{N}$ with $V(N)=T$. Then $G\left(\tau_{T}\right)$ is a star graph if and only if $\bar{M}_{1}$ is a simple module and $\bar{M}_{2}$ is a prime module for some idempotent $e \in R$.

Proof The necessity is clear by Proposition 3.1(a). For the converse, assume that $\bar{M}=\bar{M}_{1} \oplus \bar{M}_{2}$, where $\bar{M}_{1}$ is a simple module and $\bar{M}_{2}$ is a prime for some idempotent $e \in R$. Using the Proposition 3.1(b), $G\left(\tau_{T}\right)$ is a complete bipartite graph with two parts $U$ and $V$ such that $N \in U$ if and only if $V(N)=V\left(M_{1} \oplus Q_{2}\right)$ and $K \in V$ if and only if $V(K)=V\left(Q_{1} \oplus M_{2}\right)$. We claim that $|U|=1$. Otherwise, $V\left(M_{1} \oplus Q_{2}\right)=V\left(N_{1} \oplus N_{2}\right)$, where $N_{1} \leq M_{1}$ and $N_{2} \leq M_{2}$. If $N_{1} \neq M_{1}$, then $\sqrt{\left(N_{1}: M_{1}\right) M_{1}}=M_{1}$, a contradiction (note that if $M$ is a multiplication module or a primeful module, then $\sqrt{(N: M) M} \neq M$, where $N<M)$. If $N_{2} \neq Q_{2}$, then $V\left(Q_{1} \oplus N_{2}\right)=T$, a contradiction. So $G\left(\tau_{T}\right)$ is a star graph.

Theorem 3.5 If $G\left(\tau_{T}\right)$ is a tree, then $G\left(\tau_{T}\right)$ is a star graph.

Proof Suppose that $G\left(\tau_{T}\right)$ is not a star graph. Then $G\left(\tau_{T}\right)$ has at least four vertices. Obviously, there are two adjacent vertices $L$ and $K$ of $G\left(\tau_{T}\right)$ such that $|N(L) \backslash\{K\}| \geq 1$ and $|N(K) \backslash\{L\}| \geq 1$. Let $N(L) \backslash\{K\}=\left\{L_{i}\right\}_{i \in \Lambda}$ and $N(K) \backslash\{L\}=\left\{K_{j}\right\}_{j \in \Gamma}$. Since $G\left(\tau_{T}\right)$ is a tree, we have $N(L) \cap N(K)=\emptyset$. By [4, Theorem 2.10], $\operatorname{diam}\left(G\left(\tau_{T}\right)\right) \leq 3$. So every edge of $G\left(\tau_{T}\right)$ is of the form $\{L, K\},\left\{L, L_{i}\right\}$ or $\left\{K, K_{j}\right\}$, for some $i \in \Lambda$ and $j \in \Gamma$. Now, Pick $p \in \Lambda$ and $q \in \Gamma$. Since $G\left(\tau_{T}\right)$ is a tree, $L_{p} K_{q}$ is a vertex of $G\left(\tau_{T}\right)$. If $L_{p} K_{q}=L_{u}$ for some $u \in \Lambda$, then $V\left(K L_{u}\right)=T$, a contradiction. If $L_{p} K_{q}=K_{v}$, for some $v \in \Gamma$, then $V\left(L K_{v}\right)=T$, a contradiction. If $L_{p} K_{q}=L$ or $L_{p} K_{q}=K$, then $V\left(L^{2}\right)=T$ or $V\left(K^{2}\right)=T$, respectively, and hence $V(L)=T$ or $V(K)=T$, a contradiction. So the claim is proved.

Theorem 3.6 Let $R$ be an Artinian ring, $M$ be a multiplication or a primeful module, and suppose that $\bar{M}$ does not have a non-zero submodule $\bar{F} \neq \bar{N}$ with $V(N)=T$. If $G\left(\tau_{T}\right)$ is a bipartite graph, then $|T|=2$ and $G\left(\tau_{T}\right) \cong K_{2}$.

Proof At first we recall that if $G\left(\tau_{T}\right) \neq \emptyset$, then $\left|E\left(G\left(\tau_{T}\right)\right)\right| \geq 1$. Assume that $G\left(\tau_{T}\right)$ is a bipartite graph. Therefore $G\left(\tau_{T}\right)$ is not empty. We show that $R$ can not be a local ring. Otherwise, $m$ is the unique maximal ideal of $R$ and hence is the unique prime ideal. Then [14, Corollary 2.11] implies that $m M$ is the only prime submodule of $M$ so that $G\left(\tau_{T}\right)=\emptyset$, a contradiction. Hence by [8, Theorem 8.7], $R=R_{1} \oplus \ldots \oplus R_{n}$, where $R_{i}$ is an Artinian local ring for $i=1, \ldots, n$ and $n \geq 2$. By Lemma 2.2 and Proposition 2.3, since $G\left(\tau_{T}\right)$ is a bipartite graph, we have $n=2$ and hence $\bar{M} \cong \bar{M}_{1} \oplus \bar{M}_{2}$ for some idempotent $e \in R$ (for example, if $n=3$, then $M_{1} \oplus Q_{2} \oplus Q_{3}, Q_{1} \oplus M_{2} \oplus Q_{3}$, and $Q_{1} \oplus Q_{2} \oplus M_{3}$ form a triangle that is a contradiction). By Lemma 3.3, $\bar{M}_{1}$ and $\bar{M}_{2}$ are prime modules. Then it is easy to see that $\bar{M}_{1}$ and $\bar{M}_{2}$ are vector spaces over $R / \operatorname{Ann}\left(\bar{M}_{1}\right)$ and $R / \operatorname{Ann}\left(\bar{M}_{2}\right)$, respectively and so are semisimple $R$-modules. Since $G\left(\tau_{T}\right)$ is a bipartite graph, $\bar{M}_{1}$ and $\bar{M}_{2}$ are simple $R$-modules. A Similar argument as we did in proof of Corollary 3.4 implies that $T=\left\{M_{1} \oplus Q_{2}, Q_{1} \oplus M_{2}\right\}$ and $G\left(\tau_{T}\right) \cong K_{2}$.

Proposition 3.7 Assume that $M$ is a multiplication module, $\operatorname{Ann}(\bar{M})$ is a nil ideal, and $\bar{M}$ does not have a non-zero submodule $\bar{F} \neq \bar{N}$ with $V(N)=T$.

(a) If $G\left(\tau_{T}\right)$ is a finite bipartite graph, then $|T|=2$ and $G\left(\tau_{T}\right) \cong K_{2}$.

(b) If $G\left(\tau_{T}\right)$ is a regular graph of finite degree, then $|T|=2$ and $G\left(\tau_{T}\right) \cong K_{2}$.

Proof (a) By Theorem 2.11, $\bar{M}$ is an Artinian and Noetherian module so that $R / \operatorname{Ann}(\bar{M})$ is an Artinian ring. A similar arguments in Theorem 3.6 says that, $R / \operatorname{Ann}(\bar{M})$ is a non-local ring. So by [8, Theorem 8.7] and Lemma 2.2, there exist pairwise orthogonal idempotents modulo Ann $(\bar{M})$. By lemma $2.4, \bar{M} \cong \bar{M}_{1} \oplus \bar{M}_{2}$, for some idempotent $e$ of $R$. Now, the proof that $G\left(\tau_{T}\right) \cong K_{2}$ is similar to the proof of Theorem 3.6.

(b) We may assume that $G\left(\tau_{T}\right)$ is not empty. So $F$ is not a prime submodule by [4, Remark 2.6] and hence there exist $r \in R$ and $m \in M$ such that $r m \in F$ but $m \notin F$ and $r \notin(F: M)$. A similar manner in proof of 
Theorem 2.11, shows that if the set of $R$-submodules of $\overline{r M}$ (resp. $(\overline{0}: \bar{M} r)$ is infinite, then $(\overline{0}: \bar{M} r)$ (resp. $\overline{r M})$ has infinite degree, a contradiction. Thus $\overline{r M}$ and $\left(\overline{0}:_{\bar{M}} r\right)$ have finite length so that $\bar{M}$ has a finite length. Therefore $R / \operatorname{Ann}(\bar{M})$ is an Artinian ring. As in the proof of part (a), $\bar{M} \cong \bar{M}_{1} \oplus \bar{M}_{2}$ for some idempotent $e \in R$. If $\bar{M}_{1}$ has one non-trivial submodule $\bar{N}$, then $\operatorname{deg}\left(Q_{1} \oplus M_{2}\right)>\operatorname{deg}\left(N \oplus M_{2}\right)$ (we note that by [6, Proposition 2.5], $\bar{N} \bar{K}=(\overline{0})$ for some $\left.(\overline{0}) \neq \bar{K}<\bar{M}_{1}\right)$ and this contradicts the regularity of $G\left(\tau_{T}\right)$. Hence $\bar{M}_{1}$ is a simple module. Similarly, $\bar{M}_{2}$ is a simple module. Finally a similar argument as we have seen in Theorem 3.6 gives $G\left(\tau_{T}\right) \cong K_{2}$.

\section{Coloring of the Zariski-topology graph of modules}

The purpose of this section is to study the coloring of the Zariski topology-graph of modules and investigate the interplay between $\chi\left(G\left(\tau_{T}\right)\right)$ and $\omega\left(G\left(\tau_{T}\right)\right)$. We note that since $E\left(G\left(\tau_{T}\right)\right) \geq 1$ when $G\left(\tau_{T}\right) \neq \emptyset$, then $\left.\chi\left(G\left(\tau_{T}\right)\right)\right) \geq 2$.

Theorem 4.1 Let $\bar{M}$ be an Artinian module such that for every minimal submodule $\bar{N}$ of $\bar{M}, N$ is a vertex in $G\left(\tau_{T}\right)$. Then $\omega\left(G\left(\tau_{T}\right)\right)=\chi\left(G\left(\tau_{T}\right)\right)$.

Proof $\bar{M}$ is Artinian, so it contains a minimal submodule. Since for every minimal submodule $\bar{N}$ of $\bar{M}, N$ is a vertex in $G\left(\tau_{T}\right)$, we have $V(N) \neq T$. Also, $N \cap L=Q$, where $\bar{N}$ and $\bar{L}$ are minimal submodules of $\bar{M}$. It follows that $N$ and $L$ are adjacent in $G\left(\tau_{T}\right)$, where $\bar{N}$ and $\bar{L}$ are minimal submodules of $\bar{M}$. First, suppose that $\bar{M}$ has infinitely many minimal submodules. Then $\omega\left(G\left(\tau_{T}\right)\right)=\infty$ and there is nothing to prove. Next, assume that $\bar{M}$ has $k$ minimal submodules, where $k$ is finite. We conclude that $\chi\left(G\left(\tau_{T}\right)\right)=k=\omega\left(G\left(\tau_{T}\right)\right)$. Obviously, $\omega\left(G\left(\tau_{T}\right)\right) \geq k$. If possible, assume that $\omega\left(G\left(\tau_{T}\right)\right)>k$. Let $\Sigma=\left\{N_{\lambda}\right\}_{\lambda \in I}$, where $|I|=\omega\left(G\left(\tau_{T}\right)\right)$ be a maximum clique in $G\left(\tau_{T}\right)$. As for every $N_{\lambda} \in \Sigma, \overline{\sqrt{\left(N_{\lambda}: M\right) M}}$ contains a minimal submodule, there exists a minimal submodule $\bar{K}$ and submodules $N_{i}$ and $N_{j}$ in $\Sigma$, such that $\bar{K} \subset \overline{\sqrt{\left(N_{i}: M\right) M}} \cap \overline{\sqrt{\left(N_{j}: M\right) M}}$, and hence $V(K)=T$, a contradiction. Hence $\omega\left(G\left(\tau_{T}\right)\right)=k$. Next, we claim that $G\left(\tau_{T}\right)$ is $k$-colorable. In order to prove, put $A=\left\{\bar{K}_{1}, \ldots, \bar{K}_{k}\right\}$ be the set of all minimal submodules of $\bar{M}$. Now, we define a coloring $f$ on $G\left(\tau_{T}\right)$ by setting $f(N)=\min \left\{i \mid K_{i} \subseteq \sqrt{(N: M) M}\right\}$ for every vertex $N$ of $G\left(\tau_{T}\right)$. Let $N$ and $L$ be adjacent in $G\left(\tau_{T}\right)$ and $f(N)=f(L)=j$. Thus $K_{j} \subseteq \sqrt{(N: M) M} \cap \sqrt{(L: M) M}$, a contradiction. It implies that $f$ is a proper $k$ coloring of $G\left(\tau_{T}\right)$ and hence $\chi\left(G\left(\tau_{T}\right)\right) \leq k=\omega\left(G\left(\tau_{T}\right)\right)$, as desired.

Theorem 4.2 Assume that $\bar{M}$ is a faithful module. Then the following statements are equivalent.

(a) $\chi\left(G\left(\tau_{\operatorname{Spec}(M)}\right)\right)=2$.

(b) $G\left(\tau_{\mathrm{Spec}(M)}\right)$ is a bipartite graph with two non-empty parts.

(c) $G\left(\tau_{\mathrm{Spec}(M)}\right)$ is a complete bipartite graph with two non-empty parts.

(d) Either $R$ is a reduced ring with exactly two minimal prime ideals or $G\left(\tau_{\operatorname{Spec}(M)}\right)$ is a star graph with more than one vertex.

Proof By using Lemma 2.8, $G\left(\tau_{\operatorname{Spec}(M)}\right)$ and $A G(M)^{*}$ are the same and so [5, Theorem 3.3] completes the proof.

Lemma 4.3 Assume that $T$ is a finite set. Then $\left.\chi\left(G\left(\tau_{T}\right)\right)\right)$ is finite. In particular, $\left.\omega\left(G\left(\tau_{T}\right)\right)\right)$ is finite.

Proof Suppose that $T=\left\{P_{1}, P_{2}, \ldots, P_{k}\right\}$ is a finite set of distinct prime submodules of $M$. Define a coloring $f(N)=\min \left\{n \in \mathbb{N} \mid P_{n} \notin V(N)\right\}$, where $N$ is a vertex of $G\left(\tau_{T}\right)$. We can see that $\left.\chi\left(G\left(\tau_{T}\right)\right)\right) \leq k$.

Theorem 4.4 For every module $M, \omega\left(G\left(\tau_{T}\right)\right)=2$ if and only if $\chi\left(G\left(\tau_{T}\right)\right)=2$. In particular, $G\left(\tau_{T}\right)$ is bipartite if and only if $G\left(\tau_{T}\right)$ is triangle-free.

Proof Let $\omega\left(G\left(\tau_{T}\right)\right)=2$. On the contrary assume that $G\left(\tau_{T}\right)$ is not bipartite. So $G\left(\tau_{T}\right)$ contains an odd cycle. Suppose that $C:=N_{1}-N_{2}-\cdots-N_{2 k+1}-N_{1}$ be a shortest odd cycle in $G\left(\tau_{T}\right)$ for some natural number $k$. Clearly, $k \geq 2$. Since $C$ is a shortest odd cycle in $G\left(\tau_{T}\right), N_{3} N_{2 k+1}$ is a vertex. Now consider the vertices $N_{1}, N_{2}$, and $N_{3} N_{2 k+1}$. If $N_{1}=N_{3} N_{2 k+1}$, then $V\left(N_{4} N_{1}\right)=T$. This implies that $N_{1}-N_{4}-\cdots-N_{2 k+1}-N_{1}$ is an odd cycle, a contradiction. Thus $N_{1} \neq N_{3} N_{2 k+1}$. If $N_{2}=N_{3} N_{2 k+1}$, then we have $C_{3}=N_{2}-N_{3}-N_{4}-N_{2}$, again a contradiction. Hence $N_{2} \neq N_{3} N_{2 k+1}$. It is easy to check $N_{1}, N_{2}$, and $N_{3} N_{2 k+1}$ form a triangle in $G\left(\tau_{T}\right)$, a contradiction. The converse is clear. In particular, we note that empty graphs are bipartite graphs. $\square$ 
Corollary 4.5 Assume that $e \in R$ is an idempotent element and $\bar{M}$ does not have a non-zero submodule $\bar{F} \neq \bar{N}$ with $V(N)=T$. Then $G\left(\tau_{T}\right)$ is a complete bipartite graph if and only if $\bar{M}_{1}$ and $\bar{M}_{2}$ are prime modules.

Proof Assume that $G\left(\tau_{T}\right)$ is a complete bipartite graph. Therefore Theorem 4.4 states that $G\left(\tau_{T}\right)$ is a trianglefree graph. So Lemma 3.3 follows that $\bar{M}_{1}$ and $\bar{M}_{2}$ are prime modules. The conversely holds by Proposition 3.1(b).

Remark 4.6 Assume that $S$ is a multiplicatively closed subset of $R$ such that $S \cap\left(\cup_{P \in T}(P: M)\right)=\emptyset$. Let $T_{S}=\left\{S^{-1} P \mid P \in T\right\}$. One can see that $V(N)=T$ if and only if $V\left(S^{-1} N\right)=T_{S}$, where $M$ is a finitely generated module.

Theorem 4.7 Let $S$ be a multiplicatively closed subset of $R$ defined as in Remark 4.6 and $M$ is a finitely generated module. Then $G\left(\tau_{T_{S}}\right)$ is a retract of $G\left(\tau_{T}\right)$ and $\omega\left(G\left(\tau_{T_{S}}\right)\right)=\omega\left(G\left(\tau_{T}\right)\right)$.

Proof Consider a vertex map $\phi: V\left(G\left(\tau_{T}\right)\right) \longrightarrow V\left(G\left(\tau_{T_{S}}\right)\right), N \longrightarrow N_{S}$. Clearly, $N_{S} \neq K_{S}$ implies that $N \neq K$ and $V(N) \cup V(K)=T$ if and only if $V\left(N_{S}\right) \cup V\left(K_{S}\right)=T_{S}$. Thus $\phi$ is surjective and hence $\omega\left(G\left(\tau_{T_{S}}\right)\right) \leq \omega\left(G\left(\tau_{T}\right)\right)$. If $N \neq K$ and $V(N) \cup V(K)=T$, then we show that $N_{S} \neq K_{S}$. On the contrary suppose that $N_{S}=K_{S}$. Then $V\left(N_{S}^{2}\right)=V\left(N_{S} K_{S}\right)=V\left(N_{S}\right) \cup V\left(K_{S}\right)=T_{S}$ and so $V\left(N^{2}\right)=T$, a contradiction. This shows that the map $\phi$ is a graph homomorphism. Now, for any vertex $N_{S}$ of $G\left(\tau_{T_{S}}\right)$, we can choose a fixed vertex $N$ of $G\left(\tau_{T}\right)$. Then $\phi$ is a retract (graph) homomorphism which clearly implies that $\omega\left(G\left(\tau_{T_{S}}\right)\right)=\omega\left(G\left(\tau_{T}\right)\right)$ under the assumption.

Corollary 4.8 Let $S$ be a multiplicatively closed subset of $R$ defined as in Remark 4.6 and let $M$ be a finitely generated module. Then $\chi\left(A G\left(M_{S}\right)\right)=\chi(A G(M))$.

Corollary 4.9 Assume that $M$ is a semiprime module and $A G(M)^{*}$ does not have an infinite clique. Then $M$ is a faithful module and $0=\left(P_{1} \cap \ldots \cap P_{k}: M\right)$, where $P_{i}$ is a prime submodule of $M$ for $i=1, \ldots, k$.

Proof By [5, Theorem 3.8 (b)], $M$ is a faithful module and the last assertion follows directly from the proof of [5, Theorem $3.8(\mathrm{~b})]$.

Recall that the girth of a graph $G$ is the length of a shortest cycle in $G$ and denoted by $\operatorname{gr}(G)$.

Proposition 4.10 Let $R$ be an Artinian ring, $\bar{M}$ be a multiplication module, and let $T$ be a closed subset of $\operatorname{Spec}(M)$. Then we have the following statements.

(a) If $S$ is a finite subset of $T$, then there exists a clique of size $|S|$ in $G\left(\tau_{T}\right)$.

(b) We have $\omega\left(G\left(\tau_{T}\right)\right) \geq|\operatorname{Min}(T)|$ and if $|\operatorname{Min}(T)| \geq 3$, then $\operatorname{gr}\left(G\left(\tau_{T}\right)\right)=3$.

(c) If $\sqrt{(\overline{0})}=(\overline{0})$, then $\chi\left(G\left(\tau_{\operatorname{Spec}(M)}\right)\right)=\omega\left(G\left(\tau_{\operatorname{Spec}(M)}\right)\right)=|\operatorname{Min}(T)|$.

Proof (a) Let $R$ be an Artinian ring and let $\bar{M}$ be a multiplication module. Then [14, Corollary 2.9] implies that $\bar{M}$ is a cyclic module. We show that $T=\operatorname{Min}(T)$. Suppose that $P_{1} \subseteq P_{2}$, where $P_{1}, P_{2} \in T$. Then $\left(P_{1}: M\right)=\left(P_{2}: M\right)$ because every prime ideal in $R$ is maximal. Since $\bar{M}$ is multiplication, we have $P_{1}=P_{2}$ and finally the proof is straightforward by the facts that $A G(\bar{M})=A G(\bar{M})^{*}$, [6, Theorem 3.6], and $A G(\bar{M})$ is isomorphic with a subgraph of $G\left(\tau_{T}\right)$ by Lemma 2.8 .

(b) This is clear by item (a).

(c) If $|\operatorname{Min}(T)|=\infty$, then by part (b), there is nothing to prove. Otherwise, [6, Theorem 3.8] implies that $A G(\bar{M})$ does not have an infinite clique. So $\bar{M}$ is a faithful module by Corollary 4.9. Next, Lemma 2.8 says that $G\left(\tau_{\operatorname{Spec}(M)}\right)$ and $A G(M)^{*}$ are the same. Now the result follows by [6, Theorem 3.8].

Lemma 4.11 Assume that $\bar{M}$ is a semiprime module. Then the following statements are equivalent.

(a) $\left.\chi\left(G\left(\tau_{\operatorname{Spec}(M)}\right)\right)\right)$ is finite.

(b) $\left.\omega\left(G\left(\tau_{\operatorname{Spec}(M)}\right)\right)\right)$ is finite.

(c) $\left.G\left(\tau_{\operatorname{Spec}(M)}\right)\right)$ does not have an infinite clique.

Proof $(a) \Longrightarrow(b) \Longrightarrow(c)$ is clear.

$(c) \Longrightarrow(a)$ Suppose that $\left.G\left(\tau_{\operatorname{Spec}(M)}\right)\right)$ does not have an infinite clique. By Lemma 2.8, $A G(\bar{M})^{*}$ does not have an infinite clique and so by Corollary 4.9, there exists a finite number of prime submodules $P_{1}, \ldots, P_{k}$ of $M$ such that $(F: M)=\left(P_{1} \cap \ldots \cap P_{k}: M\right)$. Define a coloring $f(N)=\min \left\{n \in \mathbb{N} \mid P_{n} \notin V(N)\right\}$, where $N$ is a vertex of $G\left(\tau_{T}\right)$. Then we have $\left.\chi\left(G\left(\tau_{\operatorname{Spec}(M)}\right)\right)\right) \leq k$. 
Corollary 4.12 Assume that $A G(M / F)^{*}$ does not have an infinite clique. Then $G\left(\tau_{\operatorname{Spec}(M)}\right)$ and $A G(M)^{*}$ are the same. Also, $\left.\chi\left(G\left(\tau_{\operatorname{Spec}(M)}\right)\right)\right)$ is finite.

Proof Since $M / F$ is a semiprime module, by Corollary $4.9, M / F$ is a faithful module and there exists a finite number of prime submodules $P_{1}, \ldots, P_{k}$ of $M$ such that $(F: M)=\left(P_{1} \cap \ldots \cap P_{k}: M\right)$. So the result follows by Lemma 2.8 and from the proof of (c) $\Longrightarrow$ (a) of Lemma 4.11 .

We recall that $M$ is said to be $X$-injective if either $\operatorname{Spec}(M)=\emptyset$ or the natural map of $X=\operatorname{Spec}(M)$ is injective (see [7]).

Proposition 4.13 Suppose that $\sqrt{(\overline{0})}=(\overline{0})$, for every minimal member $P$ of $\operatorname{Spec}(M),(P: M)$ is a minimal ideal of $R$, and $\bar{M}$ is an $X$-injective module. Then the following statements are equivalent.

(a) $\chi\left(G\left(\tau_{\operatorname{Spec}(M)}\right)\right)$ is finite.

(b) $\omega\left(G\left(\tau_{\mathrm{Spec}(M)}\right)\right)$ is finite.

(c) $G\left(\tau_{\mathrm{Spec}(M)}\right)$ does not have an infinite clique.

(d) $\operatorname{Min}(\operatorname{Spec}(M))$ is a finite set.

Proof (a) $\Longrightarrow(\mathrm{b}) \Longrightarrow(\mathrm{c})$ is clear.

(c) $\Longrightarrow$ (d) Suppose $G\left(\tau_{\operatorname{Spec}(M)}\right)$ does not have an infinite clique. By Lemma 2.8, $A G(\bar{M})^{*}$ does not have an infinite clique and hence by Corollary 4.9, there exists a finite number of prime submodules $P_{1}, \ldots, P_{k}$ of $M$ such that $(F: M)=\left(P_{1} \cap P_{2} \cap \cdots \cap P_{k}: M\right)$. By assumptions, one can see that $\operatorname{Min}(\operatorname{Spec}(M))$ is a finite set.

(d) $\Longrightarrow$ (a) Assume that $\operatorname{Min}(\operatorname{Spec}(M))$ is a finite set (equivalently, $\bar{M}$ has a finite number of minimal prime submodules) so that $(F: M)=\left(P_{1} \cap P_{2} \cap \cdots \cap P_{k}: M\right)$, where $\operatorname{Min}(\operatorname{Spec}(M))=\left\{P_{1}, \ldots, P_{k}\right\}$. Define a coloring $f(N)=\min \left\{n \in N \mid P_{n} \notin V(N)\right\}$, where $N$ is a vertex of $G\left(\tau_{\operatorname{Spec}(M)}\right)$. Then we have $\chi\left(G\left(\tau_{\operatorname{Spec}(M)}\right)\right) \leq k$.

Example 4.14 If $M$ is a faithfully flat $R$-module (for example, free modules), then $p M$ is a $p$-prime submodule of $M$, where $p$ is a prime ideal of $R$ by [10, Theorem 3]. So for every minimal prime submodule $P$ of $M$, $(P: M)$ is a minimal ideal of $R$.

Proposition 4.15 Assume that $\sqrt{(\overline{0})}=(\overline{0})$ and $\bar{M}$ is a faithful module. Then the following statements are equivalent.

(a) $\chi\left(G\left(\tau_{\operatorname{Spec}(M)}\right)\right)$ is finite.

(b) $\omega\left(G\left(\tau_{\mathrm{Spec}(M)}\right)\right)$ is finite.

(c) $G\left(\tau_{\mathrm{Spec}(M)}\right)$ does not have an infinite clique.

(d) $R$ has a finite number of minimal prime ideals.

(e) $\chi\left(G\left(\tau_{\operatorname{Spec}(M)}\right)\right)=\omega\left(G\left(\tau_{\operatorname{Spec}(M)}\right)\right)=|\operatorname{Min}(R)|=k$, where $k$ is finite.

Proof This is clear by Lemma 2.8, [5, Proposition 3.10], and [5, Corollary 3.11].

Acknowledgements The authors express their deep gratitude to learned referees for their meticulous reading, contributions and valuable suggestions which have definitely improved the manuscript. This research was in part supported by a grant from IPM (No. 96130028).

Open Access This article is licensed under a Creative Commons Attribution 4.0 International License, which permits use, sharing, adaptation, distribution and reproduction in any medium or format, as long as you give appropriate credit to the original author(s) and the source, provide a link to the Creative Commons licence, and indicate if changes were made. The images or other third party material in this article are included in the article's Creative Commons licence, unless indicated otherwise in a credit line to the material. If material is not included in the article's Creative Commons licence and your intended use is not permitted by statutory regulation or exceeds the permitted use, you will need to obtain permission directly from the copyright holder. To view a copy of this licence, visit http://creativecommons.org/licenses/by/4.0/.

\section{References}

1. Anderson, D.F.; Livingston, P.S.: The zero-divisor graph of a commutative ring. J. Algebra 217, 434-447 (1999)

2. Anderson, W.; Fuller, K.R.: Rings and Categories of Modules. Springer, New York (1974)

3. Ansari-Toroghy, H.; Farshadifar, F.: Product and dual product of submodules. Far East J. Math. Sci 25(3), 447-455 (2008)

4. Ansari-Toroghy, H.; Habibi, S.: The Zariski topology-graph of modules over commutative rings. Commun. Algebra 42, 3283-3296 (2014) 
5. Ansari-Toroghy, H.; Habibi, S.: The annihilating-submodule graph of modules over commutative rings. Math. Rep. 20(70), 245-262 (2018)

6. Ansari-Toroghy, H.; Habibi, S.: The annihilating-submodule graph of modules over commutative rings II. Arab. J. Math. 5, 187-194 (2016)

7. Ansari-Toroghy, H.; Ovlyaee-Sarmazdeh, R.: On the prime spectrum of X-injective modules. Commun. Algebra 38, 2606$2621(2010)$

8. Atiyah, M.F.; Macdonald, I.G.: Introduction to Commutative Algebra. Addison-Wesley, Boston (1969)

9. Beck, I.: Coloring of commutative rings. J. Algebra 116, 208-226 (1988)

10. Chin-Pi, L.: Prime submodules of modules. Comment. Math. Univ. St. Pauli 33(1), 61-69 (1984)

11. Chin-Pi, L.: The Zariski topology on the prime spectrum of a module. Houst. J. Math. 25(3), 417-432 (1999)

12. Chin-Pi, L.: A module whose prime spectrum has the surjective natural map. Houst. J. Math 33(1), 125-143 (2007)

13. Diestel, R.: Graph theory. In: Grad, Texts in Math. Springer (2005)

14. Elbast, Z.A.; Smith, P.F.: Multiplication modules. Commun. Algebra 16, 755-779 (1988)

15. Lam, T.Y.: A First Course in Non-commutative Rings. Springer, New York (1991)

16. McCasland, R.L.; Moor, M.E.: Prime submodules. Commun. Algebra 20(6), 1803-1817 (1992)

17. Tavallaee, H.A.; Varmazyar, R.: Semi-radicals of submodules in modules. IUST Int. J. Eng. Sci. 19, 21-27 (2008)

Publisher's Note Springer Nature remains neutral with regard to jurisdictional claims in published maps and institutional affiliations. 